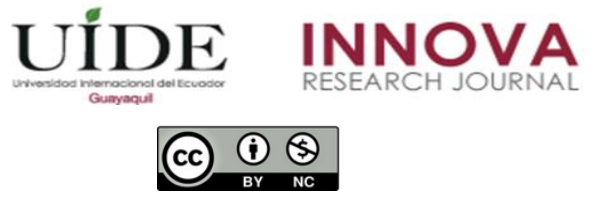

INNOVA Research Journal, ISSN 2477-9024

(Septiembre-Diciembre 2020). Vol. 5, No.3.2 pp. 1-18

DOI: https://doi.org/10.33890/innova.v5.n3.2.2020.1601

URL: http://revistas.uide.edu.ec/index.php/innova/index

Correo: innova@uide.edu.ec

\title{
Entorno virtual sincrónico y su efecto en el desarrollo de un programa para padres y madres en tiempos de COVID-19
}

\section{Synchronous virtual environment and its effect in the development of a program for parents and mothers in times of COVID-19}

Miguel Ángel Tupac Yupanqui Bustamante

(D) https://orcid.org/0000-0003-4684-3540

Consultor Independiente, Perú

Lutgarda Palomino Gonzales

(D) https://orcid.org/0000-0002-5948-341X

Carmen Ríos Espinoza

(D) https://orcid.org/0000-0001-5274-7243

María Elena Cornejo Guevara

(D) https://orcid.org/0000-0003-4235-941X

Universidad César Vallejo, Perú

Autor para correspondencia: miguelangel.tyb@gmail.com; luupago14@gmail.com; carmen_rios_9@hotmail.com; ma.elena_2624@hotmail.com

Fecha de recepción: 06 de octubre de 2020 - Fecha de aceptación: 11 de diciembre de 2020

\section{Resumen}

La presente investigación estuvo basada en la ejecución de un programa para padres y madres de niños en edad preescolar durante el confinamiento, caracterizado por el uso del entorno sincrónico como medio para interactuar, compartir las vivencias, experiencias, estrategias y recomendaciones necesarias para mejorar su convivencia familiar. El estudio tiene un enfoque cuantitativo, tipo aplicada, diseño cuasi-experimental, la muestra estuvo conformada por 80 padres y madres con hijos en edad preescolar, de los cuales 40 conformaron el grupo experimental y 40 el grupo control. El método utilizado fue de tipo no probabilístico por conveniencia. Se elaboró un Registro de observación; el cual contó con 20 ítems, posee una confiabilidad Alpha de Cronbach de 0.80. Se concluye que el entorno virtual sincrónico influye significativamente en el desarrollo del programa madres y padres en tiempos de COVID-19.

Palabras claves: entorno virtual; sincrónico; programa para padres y madres; confinamiento; COVID-19.

\footnotetext{
Abstract

The present research was based on the execution of a program for parents of preschool children during the confinement, characterized by the use of the synchronous environment as a means to
} 
interact, share experiences, experiences, strategies and recommendations necessary to improve their coexistence family. The study has a quantitative approach, applied type, quasi-experimental design, the sample consisted of 80 parents with children of preschool age, of which 40 made up the experimental group and 40 the control group. The method used was non-probabilistic for convenience. An observation record was prepared; which had 20 items, has a Cronbach's Alpha reliability of 0.80 . It is concluded that the synchronous virtual environment significantly influences the development of the mothers and fathers program in times of COVID-19.

Keywords: Virtual environment; synchronous; program for fathers and mothers; confinement $\tilde{N}$

\section{Introducción}

La familia como célula fundamental de la sociedad, cumple un rol importante para el equilibrio emocional de cada uno de sus integrantes, esta situación durante la pandemia no fue indiferente en cuanto a las repercusiones y nuevas normalidades que trajo consigo el COVID-19; dicho suceso ha generado un impacto importante en la población mundial debido al efecto negativo que ha generado en temas como la educación y sobre todo la salud. Unicef (2020) publica el informe: "Situación de familias con niños, niñas y adolescentes durante el COVID-19 en Panamá"; el estudio detalla los resultados obtenidos de 1000 hogares encuestados, encontrándose la necesidad de abordar estrategias para manejar la dinámica familiar durante el confinamiento.

La primera parte de la investigación, considera la identificación de la realidad problemática, basada en estudios e informes emitidos por instituciones de marco internacional; además, se establecen las definiciones de entorno virtual sincrónico y programas de padres y madres de familia, cada una de ellas necesarias para comprender la relevancia del estudio. Por otro lado, se establecen las principales características de las variables y como estas han podido encaminar el desarrollo de este trabajo.

El objetivo principal fue determinar la influencia del entorno virtual sincrónico en el desarrollo de un programa para padres y madres de familia en tiempos de pandemia COVID-19. El estudio muestra la articulación de las dimensiones de nuestro autor principal y las temáticas solicitadas por padres y madres de familia identificados para esta investigación, todo ello a consecuencias de las situaciones que evidenciaron en sus hijos de edad preescolar durante la pandemia.

En la segunda parte se desarrolla la teoría a partir de la revisión de estudios como el de Nuño et al. (2006), los cuales consideran que las escuelas de padres deben desarrollar temas relevantes que propicien un espacio de aprendizaje para mejorar la sana convivencia de sus miembros; además, se considera los aportes brindados por especialistas en tendencias educativas del Observatorio de Innovación Educativa del Tecnológico de Monterrey (2020) que determinan que el entorno virtual sincrónico permite la interacción en tiempo real.

Finalmente se brindarán las conclusiones y recomendaciones obtenidas de esta investigación; las cuales buscan contribuir en la comunidad académica y sociedad con estudios realizados en escenarios de pandemia. 


\section{Problemática}

A finales del mes de diciembre del 2019 y principios del 2020 se emitió una alerta mundial sobre la aparición de un virus de la familia de los coronavirus: SARS COV 2, desatando con su llegada a diversos continentes alertas de emergencia sanitaria y medidas como: distanciamiento social, uso de mascarilla, confinamiento, uso restringido de los espacios públicos, lavado de manos, entre otros. Los representantes de diversos países tomaron medidas de contingencia para intentar frenar el impacto de la propagación de este virus, frente a ello las empresas de diversos rubros apostaron por el trabajo remoto.

Instituciones internacionales e incluso centros reconocidos de investigación informaron que el virus podría ser controlado solo ante la posible presencia de una vacuna; este tipo de noticias no solo han generado incertidumbre, sino también han propiciado que cada uno de los hogares instaure entre sus miembros que la conforman, nuevas actitudes frente a la higiene para evitar el contagio. En este mismo contexto, hogares que contaban con niños en edad pre escolar, se han visto afectados porque diversos estudios han descubierto que los niños también son vulnerables, por ello, padres y madres de familia han restringido gran parte de las actividades en bien de la salud de sus hijos, sin conocer el efecto que genera tenerlos tantos días en confinamiento.

Unicef (2020) realiza una investigación en Panamá a consecuencia de la nueva realidad que venían afrontando diversos países, la cual se enfocó a identificar la situación de las familias con hijos durante el confinamiento; se encuestó a 1000 familias, encontrándose en la dimensión: conflictos en el hogar, que 1 de cada 3 presentó algún conflicto $(n=330)$ donde el 46\% informó haber tenido conflictos leves, $48 \%$ moderados y $6 \%$ fuertes; estos hallazgos demostraron que el confinamiento está generando dificultades en la convivencia, los padres y madres de familia están mostrando preocupación por las conductas que observan en sus hijos, como: pataletas, desorganización, dificultades para conciliar el sueño y problemas alimenticos; todos estos comportamientos se han convertido en una causal que los induce a buscar información con especialistas para sobrellevar el día a día en esta nueva realidad.

El Perú no es ajeno a las repercusiones que trae consigo el confinamiento, por ello los especialistas de Unicef Perú (2020) difunden a través de su plataformas oficial seminarios virtuales para padres y madres, en los que se abordaron temáticas vinculadas al control de emociones a fin de fortalecer los lazos de amor y respeto durante la nueva convivencia; este tipo de propuestas se convierten en una alternativa para aquellas familias que cuentan con conexión a internet, accediendo a eventos formativos y orientadores, en los que se brinda diversas estrategias; sin embargo, las recomendaciones son genéricas, no están dirigidas específicamente a un rango de edad; por ello, padres y madres que cuentan con niños en edad preescolar requieren de estrategias y actividades que les permitan afrontar esta nueva realidad donde el distanciamiento social y aislamiento no están a favor de las características de niños y niñas en la primera infancia.

Méndez y Näslund (2020) brindan información sobre un estudio en el cual participaron y fue realizado entre el Banco Interamericano de desarrollo, Ministerio de Educación del Perú y Innovations for Poverty Action en 2020, donde se desarrolló una encuesta dirigida a directores, 
docentes y padres de familia de Perú, la cual permitió identificar las percepciones de cómo se está llevando a cabo la estrategia de aprendizaje remota: aprendo en casa; con la finalidad de detectar algunas falencias en el servicio educativo que se viene brindando. La encuesta establece una pregunta relacionada a la dinámica familiar: ¿qué piden las familias para mejorar la educación a distancia durante la pandemia?, la cual permitió detectar que 58\% de familias requiere que se les brinde apoyo para: estrategias de aprendizaje en casa, orientaciones familiares y materiales de aprendizaje. Los resultados permiten identificar que la familia está asumiendo un nuevo rol, ellos vienen solicitando apoyo para encaminarse en esta nueva realidad que no solo afecta la educación sino también la sana convivencia.

La Fundación Internacional de Ayuda a Niños y Adolescentes en Riesgo - ANAR (2020) difunde una publicación dirigida a padres y madres, la cual brinda consejos para afrontar la convivencia durante la proliferación de la pandemia; dentro de las recomendaciones, los especialistas sugieren que se responda de una manera adecuada los cuestionamientos relacionados con el coronavirus, explicando con claridad lo que podría estar ocurriendo y transmitir tranquilidad en todo momento. Los consejos buscan concientizar a los padres y madres sobre la importancia de controlar sus emociones frente a sus hijos durante la pandemia; los castigos físicos y verbales no son la mejor alternativa para aplicar correctivos en esta nueva realidad. El contenido difundido por organizaciones de este tipo, es sumamente necesario para manejar las eventualidades que trae consigo este nuevo contexto; sin embargo, existe una gran cantidad de padres y madres que aún no dominan plenamente el uso de las tecnologías de información, de tal forma que no acceden fácilmente a esta información profesional.

Los padres y madres de familia están asumiendo roles importantes durante el confinamiento, es por ello que para llegar a ellos se deben plantear alternativas que generen espacios de aprendizaje colectivo, en los que se aborden problemáticas y posibles soluciones que podrían manifestarse durante la nueva convivencia; una forma de llegar a ellos es trabajando escuelas para padres. Fresnillo et al. (2000) determinan que las escuelas de padres son espacios de formación para trabajar, orientar sobre las tareas y funciones parentales; además, contribuyen a la adquisición de pautas para mejorar la dinámica familiar. Las escuelas para padres y madres durante el confinamiento, se convierten en una alternativa útil que respondería al cuestionamiento que vienen aquejándolos, si bien el contexto no facilita la interacción presencial, esta propuesta podría trasladarse a entornos virtuales prácticos y adaptables a realidad.

\section{Fundamentación teórica}

La educación para adultos no solo debería enfocarse a fortalecer contenidos o desarrollar logros profesionales, debería aperturar la posibilidad de crear espacios de aprendizaje para afrontar cada etapa de la vida; el asumir el rol de padre y madre de familia, trae consigo una serie de satisfacciones y responsabilidades; no existe una guía o un manual de cómo ser padres exitosos, sin embargo, es necesario fortalecer las habilidades emocionales para poder encaminar a sus hijos. Romero (2005) considera que la sociedad continuamente presenta cambios, lo cuales influyen significativamente en las nuevas generaciones de niños, estos cambios vienen de la mano con conductas que en ocasiones no logran manejarse en el hogar, es por ello que padres y madres de familia deberían de ampliar la capacidad de entendimiento y recurrir a orientaciones con profesionales para afianzar sus roles parentales. 
Romero (2005) establece que las escuelas para para padres se originaron para atender a las necesidades familiares, con la finalidad de brindar una serie de estrategias que les permita asimilar los cambios generacionales y sobre todo comprender los problemas que se pueden presentar en cada una de las etapas que afrontan sus hijos. De la Puente (citado por Romero, 2005) afirma que: "las escuelas para padres es una estrategia importante para crear un ámbito del diálogo educativo acerca de los fines y medios de educación: porqué educamos y cómo educamos. Se inserta en el área de la comunicación o conversación, que no logra ninguna decisión operativa a corto plazo, sino solamente la reflexión, el diálogo, el consenso" (p.2). Estos aportes ratifican que el rol de los padres y madres de familia es de reinvención y actualización continua, por ello las escuelas de padres y madres de familia deben de realizarse continuamente; más aún si nos enfocamos en esta nueva realidad originada por la pandemia.

Fresnillo et al. (2000) consideran que las escuelas para padres y madres son: [...] recurso de apoyo a las familias con menores para que puedan desarrollar adecuadamente sus funciones educativas y socializadoras, y superar situaciones de necesidad y riesgo social; es uno de los programas de carácter preventivo que contribuyen a modificaciones de conductas y a la adquisición de pautas saludables de dinámica familiar (p.9).

Fresnillo et al. (2000) establecen que las escuelas para padres y madres buscan propiciar espacios de reflexión de situaciones que se presentan en la vida diaria, fortalecer la comunicación entre cada uno de los miembros de la familia, favorecer de estrategias que permitan asumir las tareas parentales de la mejor forma, detectar y afrontar las problemáticas que podrían atentar contra la estabilidad familiar. El rol que vienen desempeñando los padres durante la pandemia es arduo, la emergencia sanitaria no solo atenta contra nuestro bienestar físico, también viene amenazando la tranquilidad familiar, por ello los padres y madres de familia se encuentran en la búsqueda de información para tratar de mantener una dinámica familiar afable durante el confinamiento, recurriendo a una serie de mecanismos como: consejos familiares, consejos de amistades e incluso búsquedas en redes sociales que puedan dar soluciones prácticas a sus interrogantes; es preciso resaltar que no todas estas fuentes poseen información vertida por especialistas, por ello frente a la nueva realidad la escuelas para padres en entornos virtuales se presenta como una alternativa de aprendizaje en tiempo real.

Abordar actividades de aprendizaje con padres en tiempos de pandemia se puede convertir en una oportunidad de interacción importante, sin embargo, es un nuevo reto para especialistas, docentes y comunidad educativa, explorar las bondades y usos de la tecnología como medio para poder comunicarnos. Las familias durante el confinamiento vienen recurriendo a información para apoyar a sus hijos en la asimilación de esta nueva realidad, cada una de las familias afronta situaciones similares pero diferentes al mismo tiempo, por ello el entorno virtual debería de contar con ciertas características que permitan atender de alguna forma las consultas particulares que tienen los padres y madres de familia. En la búsqueda de soluciones se detectó que la comunicación en entornos virtuales puede darse en tiempo real a lo que se denomina: sincrónico.

Delgado (2020) considera dos tipos de aprendizaje en línea: sincrónico y asincrónico, que para efectos de este estudio se determinará aprendizaje sincrónico a la oportunidad de aprender e 
interactuar en el momento; además posee ventajas, como: interacción en tiempo real entre maestros y estudiantes, haciendo que cualquier problema o duda se resuelva en tiempo real. Vásquez (citado por Montes, Trujillo, Juaréz, Mejía y Corro, 2020) refieren que la comunicación sincrónica está dirigida al intercambio que se realiza en tiempo real entre los participantes a través de una videoconferencia, llamada, mensajería instantánea y/o sala de conversación. El entorno virtual sincrónico, es un tipo de comunicación que frente al distanciamiento social nos permite acercarnos a los demás, siendo un medio a través del cual se pueden ejecutar programas educativos, que permitan brindar orientaciones a padres y madres de familia.

García y Cuellar (2020) realizan una revisión sobre el impacto psicológico del confinamiento en la población infantil siguiendo las recomendaciones de la Organización Mundial de la Salud, en el cual se destaca el estudio realizado por Sprang y Silman en 2013 donde se indica que las puntuaciones de estrés postraumático de niños en confinamiento fueron 4 veces más altas en comparación a niños que no fueron confinados; este tipo de estudios son el punto de partida para analizar y establecer estrategias que permitan a padres y madres apoyar a sus hijos durante el aislamiento. Aguirre et al. (Citado por Gómez 2019) consideran que las escuelas para padres son un plan sistemático de formación, los cuales buscan poner en práctica actividades, de tal forma que les permitan vincularse con sus hijos. A partir de la fundamentación desarrollada en esta investigación, se implementó una escuela de padres y madres de niños y niñas en edad pre escolar en entornos virtuales sincrónicos, con la finalidad de asesorarlos y orientarlos frente a las situaciones que están afrontando con sus hijos.

Las escuelas para padres y madres de familia se convierten en la base para el desarrollo del programa "Padres talentosos", el cual estuvo dirigido a padres y madres de familia de niños y niñas en edad pre escolar. El programa se desarrolló desde el análisis de las dimensiones establecida en la investigación de: Nuño et al. (2006) los cuales establecen las siguientes dimensiones: relaciones familiares, afrontamiento de problemas, comportamiento y problemas de salud. A partir de estas dimensiones se estableció la mesa de trabajo ágil; la cual contó con la participación de: docentes de educación inicial, directoras de instituciones educativas de nivel inicial, especialista en psicología y especialista en sistemas e informática; con la finalidad de seleccionar las temáticas y espacios virtuales que permitan desarrollar la escuela de padres y madres.

El trabajo realizado entre especialistas, permitió establecer una matriz de articulación de: dimensiones, criterios y temas; los cuales se especifican en la siguiente tabla:

\section{Tabla 1}

Matriz de articulación - Programa "Padres talentosos"

\begin{tabular}{|c|c|c|}
\hline Dimensiones & Criterios & Temas \\
\hline $\begin{array}{c}\text { Afrontamiento de } \\
\text { problemas }\end{array}$ & $\begin{array}{l}\text { Búsqueda de } \\
\text { apoyo social }\end{array}$ & $\begin{array}{l}\text { TEMA } 1 \\
\text { ¿Qué es la pandemia y cómo afecta a los niños de } 3 \text { a } 6 \text { años? } \\
\text { TEMA } 2 \\
\text { ¿Cómo apoyar a niño de } 3 \text { a } 6 \text { años en sus actividades escolares } \\
\text { durante la pandemia? }\end{array}$ \\
\hline
\end{tabular}




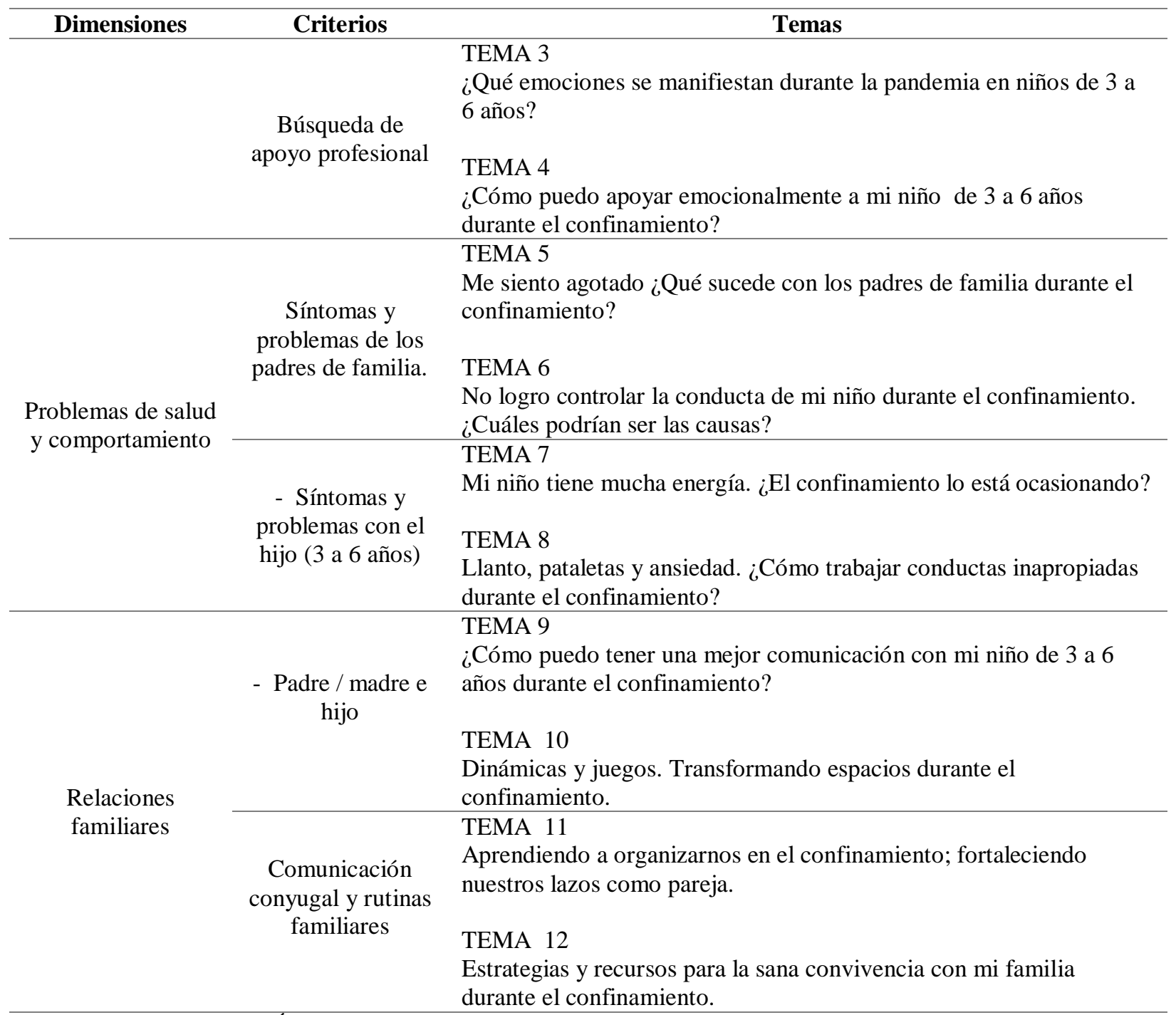

Nota: Adaptado de Nuño, Álvarez, Madrigal, Martínez y Miranda (2006)

El programa se desarrolló durante 12 sesiones, en horarios determinados a la disponibilidad de la gran mayoría de padres. La plataforma que se utilizó para desarrollar las sesiones fue ZOOM; puesto que, a opinión del especialista en sistemas e informática, contaba con mayores posibilidades para guiar a los padres en la descarga del aplicativo, acceso al link de la reunión y botones de acción para interacción en tiempo real con los especialistas.

El estudio permitió establecer como objetivo general: determinar si un entorno virtual sincrónico influye en el desarrollo del programa "Padres talentosos" en tiempos de pandemia COVID-19; además, busco determinar de manera específica si un entorno virtual sincrónico influye en el nivel de conocimiento de las dimensiones: afrontamiento del problema, relaciones familiares, problemas de salud y comportamientos desarrolladas durante la ejecución del programa "Padres talentosos". 


\section{Método}

La investigación fue de enfoque cuantitativo, tipo aplicada y cuenta con un diseño cuasiexperimental. Se diseñó un registro de observación, sometido a validez y confiabilidad; determinándose en los resultados que era aplicable. El cuestionario está compuesto por un total de 20 ítems y un formato de respuestas dicotómicas. La muestra estuvo conformada por 80 padres y madres de niños de instituciones educativas de educación básica del nivel inicial de diferentes distritos de Lima Metropolitana, el tamaño de muestra se seleccionó mediante el muestreo no probabilístico de tipo intencional: 40 padres para el grupo control y 40 para el grupo experimental a quienes se les aplicó un pretest y un postest en un solo momento.

\section{Tabla 2}

Distribución de preguntas del registro de observación

\begin{tabular}{lll}
\hline Registro de observación & Dimensiones & Preguntas \\
\hline \multirow{2}{*}{ "Padres talentosos" } & Afrontamiento del problema & $1,2,3,4,5,6,7$ \\
\cline { 2 - 3 } & $\begin{array}{l}\text { Problemas de salud y } \\
\text { comportamientos }\end{array}$ & $8,9,10,11,12,13,14$ \\
\cline { 2 - 3 } & Relaciones familiares & $15,16,17,18,19,20$ \\
\hline
\end{tabular}

Nota: Adaptado de Nuño, Álvarez, Madrigal, Martínez y Miranda (2006)

\section{Método de análisis de la información}

Los resultados estadísticos obtenidos frente a la aplicación del programa fueron los siguientes:

\section{Tabla 3}

Proceso estadístico antes y después de la implementación de un entorno virtual sincrónico para el desarrollo del programa "Padres talentosos" en tiempos de pandemia COVID-19

\begin{tabular}{|c|c|c|c|}
\hline & & $\begin{array}{l}\text { Desarrollo del programa } \\
\text { "Padres talentosos": Grupo } \\
\text { de experimental - Pretest }\end{array}$ & $\begin{array}{l}\text { Desarrollo del programa } \\
\text { "Padres talentosos": Grupo de } \\
\text { experimental - Postest }\end{array}$ \\
\hline \multirow[t]{2}{*}{$\mathrm{N}$} & Válido & 40 & 40 \\
\hline & Perdidos & 0 & 0 \\
\hline \multicolumn{2}{|l|}{ Media } & 8,9250 & 15,8750 \\
\hline \multicolumn{2}{|c|}{ Error estándar de la media } & ,26162 & ,26599 \\
\hline \multicolumn{2}{|l|}{ Mediana } & $8,9474^{\mathrm{a}}$ & $16,0526^{\mathrm{a}}$ \\
\hline \multicolumn{2}{|l|}{ Moda } & 9,00 & 17,00 \\
\hline \multicolumn{2}{|l|}{ Desviación estándar } & 1,65464 & 1,68230 \\
\hline \multicolumn{2}{|l|}{ Varianza } & 2,738 & 2,830 \\
\hline \multicolumn{2}{|l|}{ Asimetría } &, 161 &,- 610 \\
\hline
\end{tabular}




\begin{tabular}{lcc}
\hline & $\begin{array}{c}\text { Desarrollo del programa } \\
\text { "Padres talentosos": Grupo } \\
\text { de experimental - Pretest }\end{array}$ & $\begin{array}{c}\text { Desarrollo del programa } \\
\text { "Padres talentosos": Grupo de } \\
\text { experimental - Postest }\end{array}$ \\
\hline Error estándar de asimetría &, 374 &, 374 \\
Curtosis &, 120 &, 066 \\
Error estándar de curtosis &, 733 &, 733 \\
Rango & 7,00 & 7,00 \\
Mínimo & 6,00 & 12,00 \\
Máximo & 13,00 & 19,00 \\
Suma & 357,00 & 635,00 \\
\hline
\end{tabular}

a. Se ha calculado a partir de datos agrupados.

Nota. Base de datos

Según la presente tabla de frecuencia estadística, se evidencia una media de 8,923 antes del uso de un entorno virtual sincrónico para el desarrollo del programa "Padres talentosos" en tiempos de pandemia COVID-19, asimismo después de la aplicación se obtuvo una media de 15,875 , por lo tanto, se determinó que las diferencias de medias entre el pretest y postest es de manera positiva en el grupo experimental de acuerdo a la totalidad de suma.

\section{Figura 1}

Uso de un entorno virtual sincrónico para el desarrollo del programa "Padres talentosos" en tiempos de pandemia COVID-19
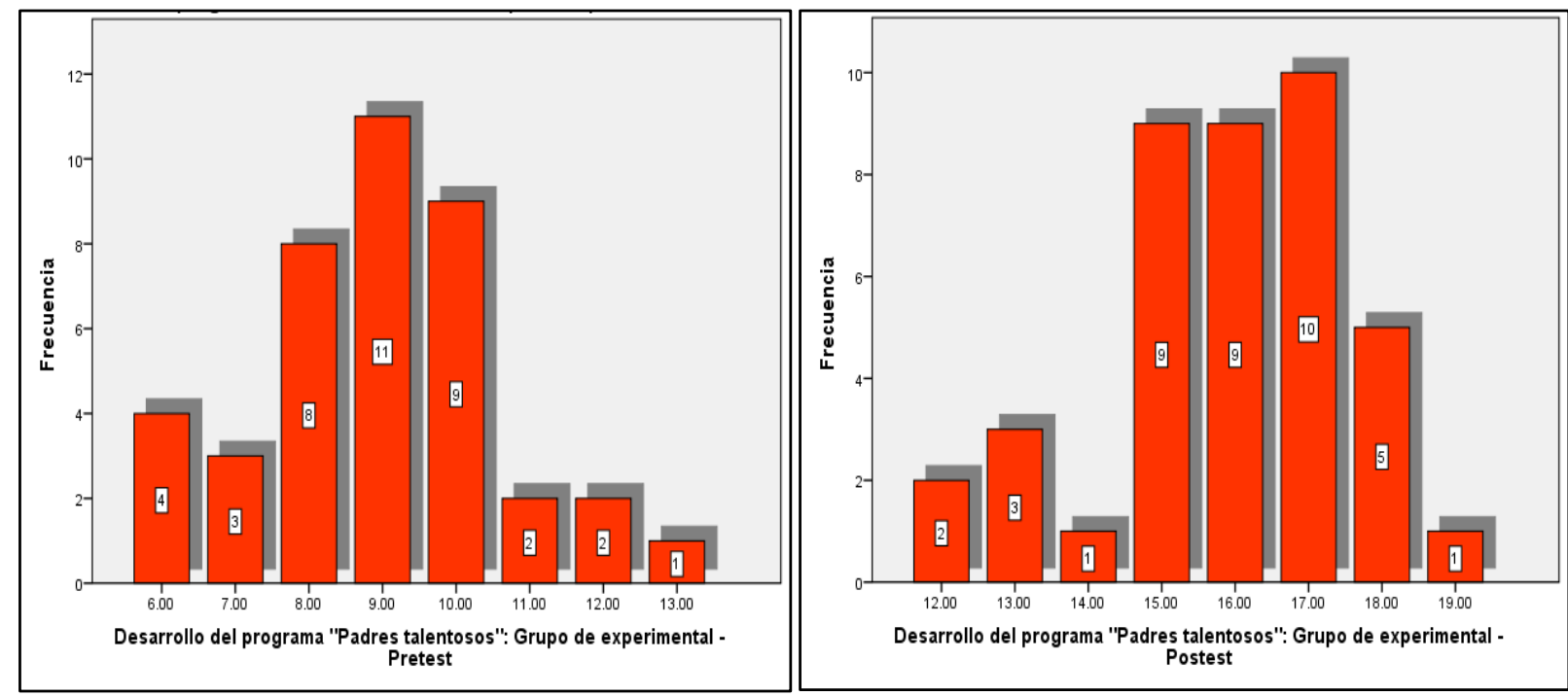

Nota. Grafica comparativa de los resultados entre el pretest y postest del grupo experimental durante el uso del entorno virtual sincrónico para el desarrollo del programa "Padres talentosos". Se observa en el eje "x" las preguntas del instrumento y en el eje " $y$ " la frecuencia de participantes por pregunta. 


\section{Tabla 4}

Proceso estadístico antes y después del uso de un entorno virtual sincrónico para la dimensión afrontamiento del problema del programa "Padres talentosos" en tiempos de pandemia COVID19

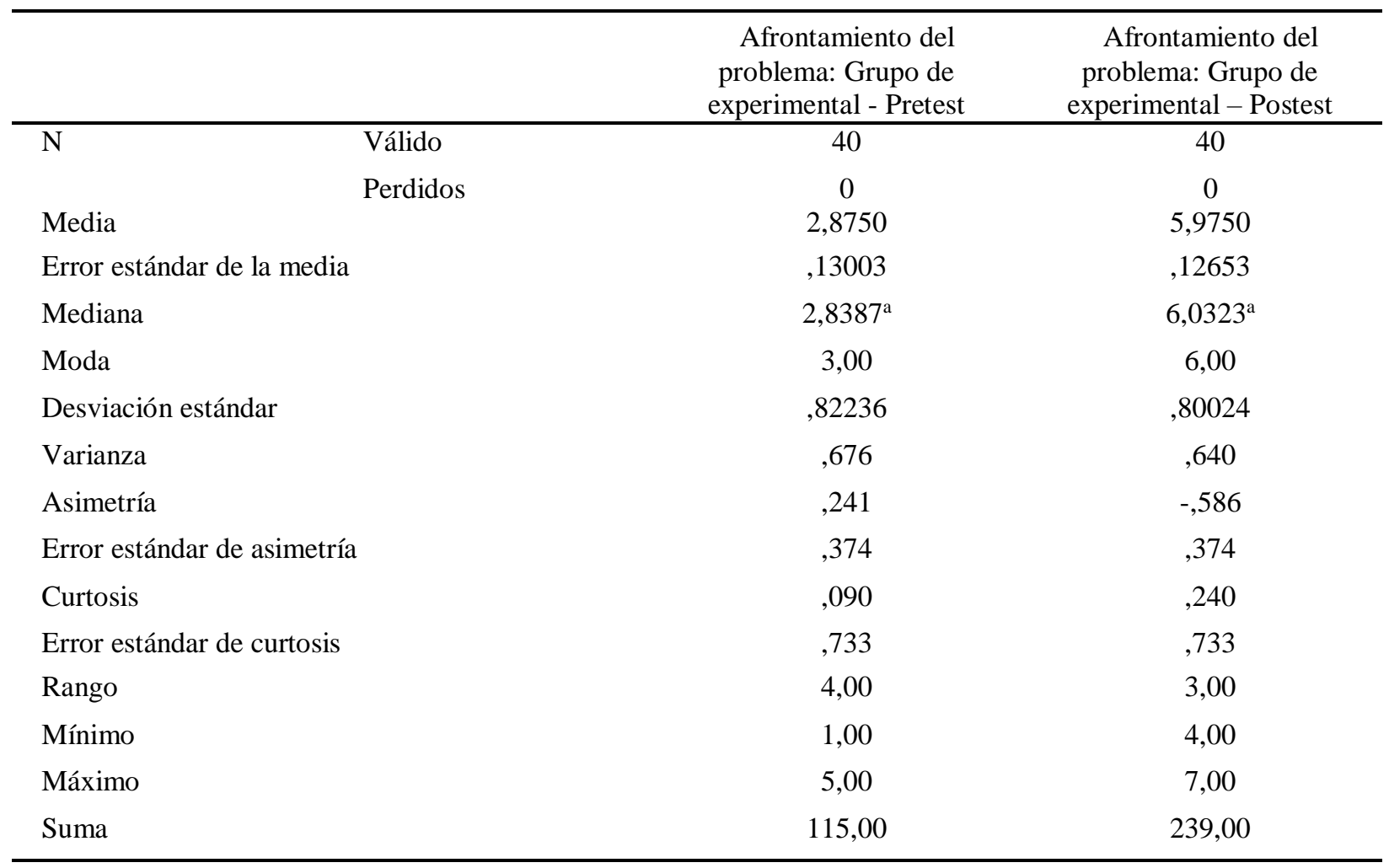

a. Se ha calculado a partir de datos agrupados.

Nota. Base de datos

Según la presente tabla de frecuencia estadística, se evidencia una media de 2,875 antes del uso de un entorno virtual sincrónico para la dimensión afrontamiento del problema en el programa "Padres talentosos" en tiempos de pandemia COVID-19, asimismo después de la aplicación se obtuvo una media de 5,975, por lo tanto, se determinó que las diferencias de medias entre el pretest y postest es de manera positiva en el grupo experimental de acuerdo a totalidad de suma. 


\section{Figura 2}

Uso de un entorno virtual sincrónico para la dimensión afrontamiento del problema del programa "Padres talentosos" en tiempos de pandemia COVID-19

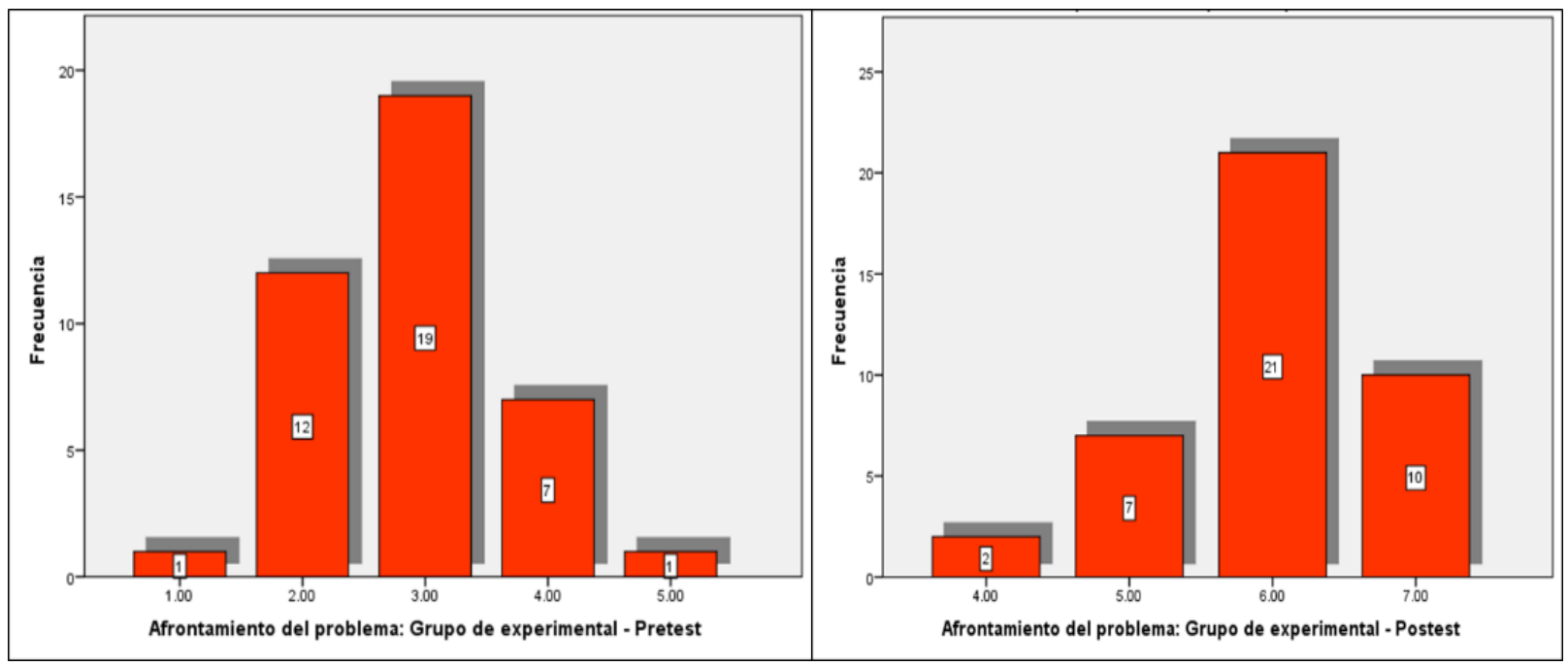

Nota. Grafica comparativa de los resultados entre el pretest y postest del grupo experimental durante el uso del entorno virtual sincrónico en la dimensión afrontamiento del problema del programa "Padres talentosos". Se observa en el eje " $x$ " las preguntas del instrumento y en el eje " $y$ " la frecuencia de participantes por pregunta.

\section{Tabla 5}

Proceso estadístico antes y después del uso de un entorno virtual sincrónico para la dimensión problemas de salud y comportamiento del programa "Padres talentosos" en tiempos de pandemia COVID-19

\begin{tabular}{|c|c|c|c|}
\hline & & $\begin{array}{c}\text { Problemas de salud y } \\
\text { comportamiento: Grupo de } \\
\text { experimental - Pretest }\end{array}$ & $\begin{array}{c}\text { Problemas de salud y } \\
\text { comportamiento: Grupo de } \\
\text { experimental - Postest }\end{array}$ \\
\hline \multirow[t]{2}{*}{$\mathrm{N}$} & Válido & 40 & 40 \\
\hline & Perdidos & 0 & 0 \\
\hline \multicolumn{2}{|c|}{ Media } & 3,3500 & 4,9250 \\
\hline \multicolumn{2}{|c|}{ Error estándar de la media } & ,20428 &, 16942 \\
\hline \multicolumn{2}{|c|}{ Mediana } & $3,1250^{\mathrm{a}}$ & $4,7778^{a}$ \\
\hline \multicolumn{2}{|c|}{ Moda } & 2,00 & 4,00 \\
\hline \multicolumn{2}{|c|}{ Desviación estándar } & 1,29199 & 1,07148 \\
\hline \multicolumn{2}{|c|}{ Varianza } & 1,669 & 1,148 \\
\hline \multicolumn{2}{|c|}{ Asimetría } &, 500 &, 551 \\
\hline \multicolumn{2}{|c|}{ Error estándar de asimetría } &, 374 & 374 \\
\hline \multicolumn{2}{|c|}{ Curtosis } & $-1,038$ &,- 726 \\
\hline \multicolumn{2}{|c|}{ Error estándar de curtosis } &, 733 &, 733 \\
\hline \multicolumn{2}{|c|}{ Rango } & 4,00 & 4,00 \\
\hline \multicolumn{2}{|c|}{ Mínimo } & 2,00 & 3,00 \\
\hline \multicolumn{2}{|c|}{ Máximo } & 6,00 & 7,00 \\
\hline \multicolumn{2}{|c|}{ Suma } & 134,00 & 197,00 \\
\hline
\end{tabular}


a. Se ha calculado a partir de datos agrupados.

Nota. Base de datos

Según la presente tabla de frecuencia estadística, se evidencia una media de 3,350 antes del uso del entorno virtual sincrónico para la dimensión problemas de salud y comportamiento del programa "Padres talentosos" en tiempos de pandemia COVID-19, asimismo después de la aplicación se obtuvo una media de 4,925, por lo tanto, se determinó que las diferencias de medias entre el pretest y postest es de manera positiva en el grupo experimental de acuerdo a totalidad de suma.

\section{Figura 3}

Uso un entorno virtual sincrónico para la dimensión problemas de salud y comportamiento del programa "Padres talentosos" en tiempos de pandemia COVID-19.

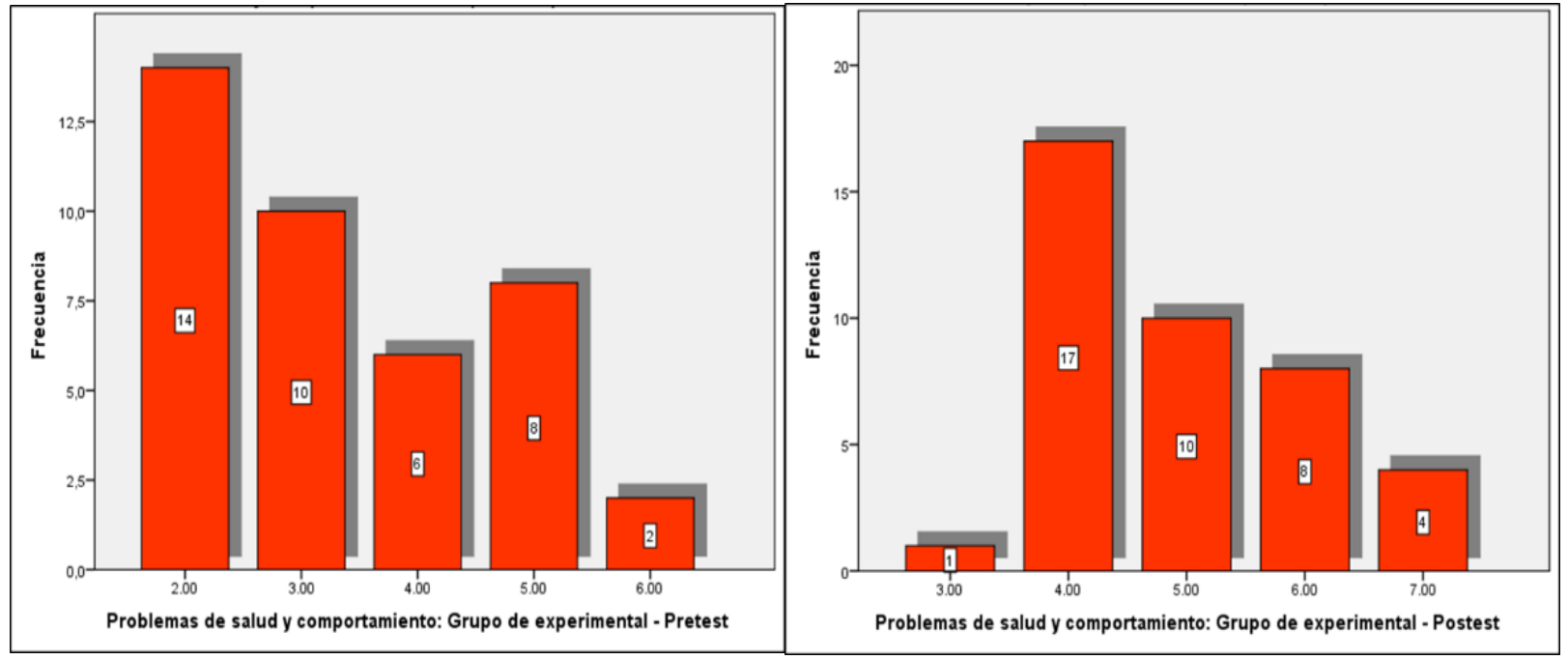

Nota. Grafica comparativa de los resultados entre el pretest y postest del grupo experimental durante el uso del entorno virtual sincrónico en la dimensión problemas de salud y comportamiento del programa "Padres talentosos". Se observa en el eje " $x$ " las preguntas del instrumento y en el eje " $y$ " la frecuencia de participantes por pregunta.

\section{Tabla 6}

Proceso estadístico antes y después del uso de un entorno virtual sincrónico para la dimensión relaciones familiares del programa "Padres talentosos" en tiempos de pandemia COVID-19

\begin{tabular}{lccc}
\hline & $\begin{array}{c}\text { Relaciones: Grupo de } \\
\text { experimental - Pretest }\end{array}$ & $\begin{array}{c}\text { Relaciones: Grupo de } \\
\text { experimental - Postest }\end{array}$ \\
\hline $\mathrm{N}$ & Válido & 40 & 40 \\
Media & 0 & 0 \\
Error estándar de la media & 2,7000 & 4,9750 \\
Mediana &, 13493 &, 17353 \\
Moda & $2,6176^{\mathrm{a}}$ & $5,1154^{\mathrm{a}}$ \\
Desviación estándar & $2,00^{\mathrm{b}}$ & 6,00 \\
&, 85335 & 1,09749
\end{tabular}




\begin{tabular}{lcc}
\hline & $\begin{array}{c}\text { Relaciones: Grupo de } \\
\text { experimental - Pretest }\end{array}$ & $\begin{array}{c}\text { Relaciones: Grupo de } \\
\text { experimental - Postest }\end{array}$ \\
\hline Varianza &, 728 & 1,204 \\
Asimetría &, 896 &,- 929 \\
Error estándar de asimetría &, 374 &, 374 \\
Curtosis & 1,104 &, 595 \\
Error estándar de curtosis &, 733 &, 733 \\
Rango & 4,00 & 4,00 \\
Mínimo & 1,00 & 2,00 \\
Máximo & 5,00 & 6,00 \\
Suma & 108,00 & 199,00 \\
\hline
\end{tabular}

a. Se ha calculado a partir de datos agrupados.

b. Existen múltiples modos. Se muestra el valor más pequeño.

Nota. Base de datos

Según la presente tabla de frecuencia estadística, se evidencia una media de 2,700 antes del uso de un entorno virtual sincrónico para la dimensión relaciones familiares del programa "Padres talentosos" en tiempos de pandemia COVID-19, asimismo después de la aplicación se obtuvo una media de 4,975, por lo tanto, se determinó que las diferencias de medias entre el pretest y postest es de manera positiva en el grupo experimental de acuerdo a totalidad de suma.

\section{Figura 4}

Uso de un entorno virtual sincrónico para la dimensión relaciones familiares del programa "Padres talentosos" en tiempos de pandemia COVID-19
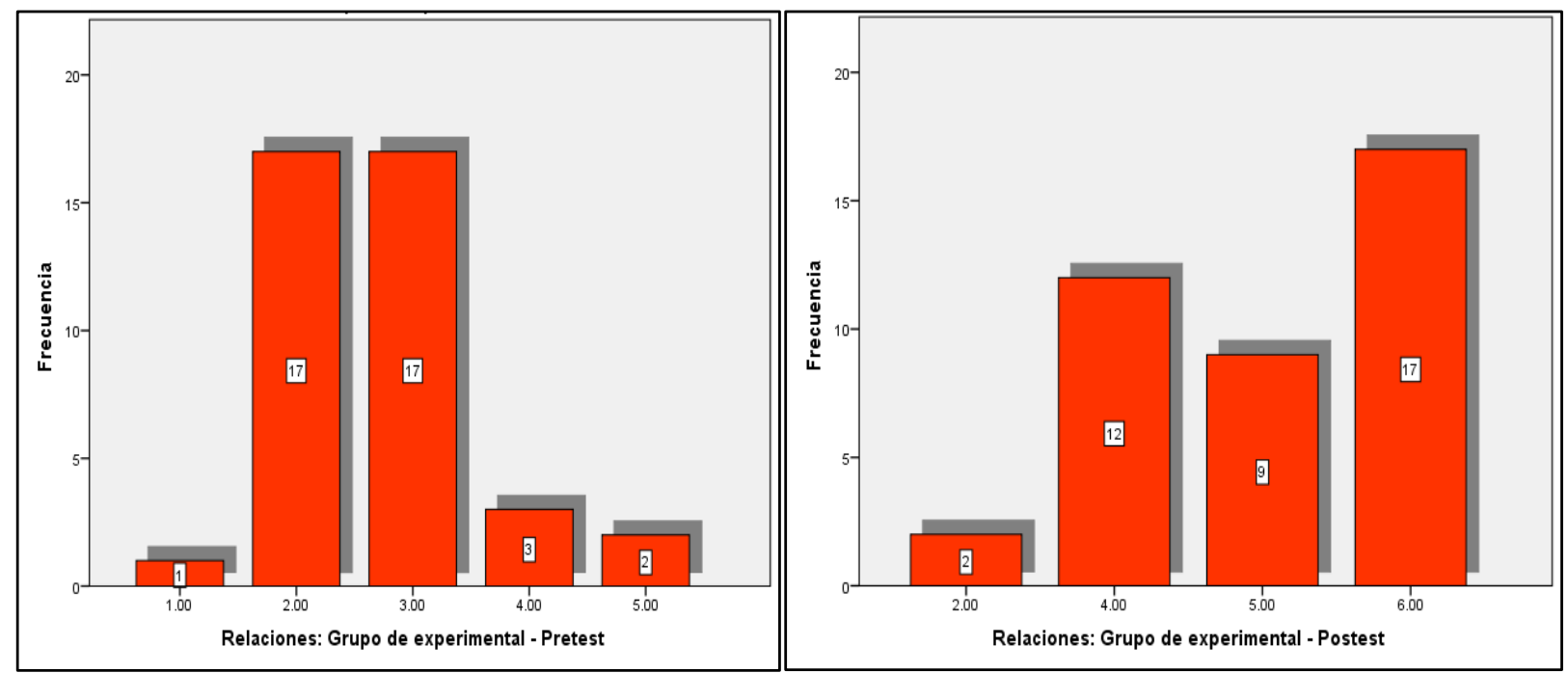

Nota. Grafica comparativa de los resultados entre el pretest y postest del grupo experimental durante el uso del entorno virtual sincrónico en la dimensión relaciones familiares del programa "Padres talentosos". Se observa en el eje " $x$ " las preguntas del instrumento y en el eje " $y$ " la frecuencia de participantes por pregunta. 


\section{Prueba de normalidad}

\section{Tabla 7}

Aplicación de la prueba de normalidad para determinar el uso del proceso estadístico para las variables y dimensiones de estudio

\begin{tabular}{|c|c|c|c|}
\hline & \multicolumn{3}{|c|}{ Shapiro-Wilk } \\
\hline & Estadístico & $\mathrm{gl}$ & Sig. \\
\hline $\begin{array}{l}\text { Afrontamiento del problema: Grupo de } \\
\text { control - Pretest }\end{array}$ & ,876 & 40 &, 000 \\
\hline Problemas de salud y comportamiento: &, 855 & 40 &, 000 \\
\hline Grupo de control - Pretest & & & \\
\hline Relaciones: Grupo de control - Pretest & ,757 & 40 &, 000 \\
\hline Desarrollo del programa "Padres & ,952 & 40 & ,091 \\
\hline talentosos": Grupo de control - Pretest & & & \\
\hline $\begin{array}{l}\text { Afrontamiento del problema: Grupo de } \\
\text { control - Postest }\end{array}$ & 889 & 40 &, 001 \\
\hline $\begin{array}{l}\text { Problemas de salud y comportamiento: } \\
\text { Grupo de control - Postest }\end{array}$ & ,889 & 40 &, 001 \\
\hline Relaciones: Grupo de control - Postest &, 874 & 40 &, 000 \\
\hline Desarrollo del programa "Padres & ,958 & 40 &, 138 \\
\hline talentosos": Grupo de control - Postest & & & \\
\hline $\begin{array}{l}\text { Afrontamiento del problema: Grupo de } \\
\text { experimental - Pretest }\end{array}$ & ,876 & 40 &, 000 \\
\hline Relaciones: Grupo de experimental - &, 828 & 40 & ,000 \\
\hline $\begin{array}{l}\text { Pretest } \\
\text { Desarrollo del programa "Padres }\end{array}$ & ,951 & 40 &, 080 \\
\hline $\begin{array}{l}\text { talentosos": Grupo de experimental - Pretest } \\
\text { Afrontamiento del problema: Grupo de } \\
\text { experimental - Postest }\end{array}$ & ,836 & 40 &, 000 \\
\hline $\begin{array}{l}\text { Problemas de salud y comportamiento: } \\
\text { Grupo de experimental - Postest }\end{array}$ & ,858 & 40 & ,000 \\
\hline $\begin{array}{l}\text { Relaciones: Grupo de experimental - } \\
\text { Postest }\end{array}$ &, 804 & 40 & ,000 \\
\hline \begin{tabular}{lcc}
\multicolumn{1}{c}{ Desarrollo } & del & programa "Padres \\
talentosos": & Grupo & de experimental - \\
Postest & &
\end{tabular} & ,932 & 40 & ,019 \\
\hline
\end{tabular}

a. Corrección de significación de Lilliefors

Nota. Base de datos.

De acuerdo a la prueba de normalidad de Shapiro-Wilk, se ha determinado que los valores estadísticos obtenidos por las variables y dimensiones no cumplen una distribución normal ( $\mathrm{p}<0.05)$, por lo tanto, corresponde utilizar la prueba estadística no paramétrica U de Mann-Whitney para muestras independientes de diferencia de medianas. 


\section{Análisis inferencial}

El proceso de verificación o contrastación fue procesado estadísticamente en el sistema (Spss) probabilidad de toma de decisiones del cumplimiento o rechazó de la hipótesis formulada.

Para la Verificación y comprobación de estableció la siguiente hipótesis general: el uso un entorno virtual sincrónico influye significativamente en el desarrollo del programa "Padres talentosos" en tiempos de pandemia COVID-19. Para efecto de la contrastación se aplicó la prueba estadística no paramétrica U de Mann-Whitney (Spss: v 24.0)

\section{Tabla 9}

Prueba estadística de U de Mann-Whitney aplicado al grupo de control y grupo experimental frente al uso de un entorno virtual sincrónico en el desarrollo del programa "Padres talentosos" en tiempos de pandemia COVID-19

\begin{tabular}{llrrr}
\hline & Padres talentosos & N & Rango promedio & Suma de rangos \\
\hline Pretest & Grupo control & 40 & 39,73 & 1589,00 \\
& Grupo experimental & 40 & 41,28 & 1651,00 \\
& Total & 80 & & \\
\cline { 2 - 5 } Postest & Grupo control & 40 & 20,75 & 830,00 \\
& Grupo experimental & 40 & 60,25 & 2410,00 \\
& Total & 80 & & \\
\hline
\end{tabular}

Estadísticos de contraste ${ }^{a}$

Desarrollo del programa "Padres talentosos":

\begin{tabular}{lcc}
\cline { 2 - 3 } & Pretest & Postest \\
\hline U de Mann-Whitney & & 10,000 \\
W de Wilcoxon & 769,000 & 830,000 \\
Z & 1589,000 & $-7,639$ \\
Sig. asintót. (bilateral) &,- 305 &, 000 \\
\hline
\end{tabular}

a. Variable de agrupación: Grupos

Nota. Base de datos.

De acuerdo a los resultados obtenidos estadísticamente en presenta tabla, según la prueba U de Mann-Whitney (prueba no paramétrica) para muestras independientes, se puede evidenciar el rango de promedio y suma de rangos una diferencia significativa entre los grupos de control y el grupo experimental del programa "Padres talentosos", obteniendo un nivel de significancia de 0,760 (Pretest) y una significancia de 0,000 (Postest) menor que $\mathrm{p}<0,05$, es decir, se rechazó la hipótesis nula (Ho) y se cumple la hipótesis alterna, concluyendo que el uso de un entorno virtual sincrónico influye significativamente en el desarrollo del programa "Padres talentosos" en tiempos de pandemia COVID-19. 


\section{Conclusiones}

Se ha demostrado a través de este estudio que el entorno virtual sincrónico influye positivamente en el desarrollo de un programa para padres de familia en tiempos de pandemia Covid 19. Por otro lado, se afirma que el uso de la tecnología está adaptándose a diversos entornos, diferentes contextos y está permitiendo acoplarse a las necesidades de las personas.

Respecto a los resultados obtenidos en cada una de las dimensiones:

Se identificó que el entorno virtual sincrónico favorece de forma significativa en la dimensión afrontamiento del problema. Los resultados muestran que el programa para padres de familia tuvo aceptación y acogida debido al tratamiento que se le dio a las temáticas relacionadas a esta dimensión y al uso del medio sincrónico como canal de interacción.

De la misma manera, se observó que el entorno virtual sincrónico favorece de forma significativa en la dimensión problemas de salud y comportamiento. Los resultados muestran que el programa para padres de familia logro abordar temáticas de interés relacionadas a esta dimensión y confirmar el uso del medio sincrónico como canal de interacción.

Finalmente, se verificó que el entorno virtual sincrónico favorece de forma significativa en la dimensión relaciones familiares. Los resultados reafirman que el programa para padres de familia en esta investigación contenía temáticas adecuadas para dicha dimensión, facilitando su comprensión; además, corrobora la influencia del uso del medio sincrónico como canal de interacción.

\section{Recomendaciones}

\section{A los directores de las instituciones educativas:}

Implementar mesas de trabajo agiles que permiten generar ideas, planes, propuestas y talleres en los cuales se puedan abordar temáticas de coyuntura que permitan brindar espacios de aprendizaje a los padres de familia.

Difundir y sensibilizar en padres de familia sobre la importancia que tiene la escuela para padres y madres en tiempos de pandemia; afianzando que este tipo de propuestas permitirá acceder a recomendaciones para afrontar las nuevas situaciones que se están presentando a consecuencia del confinamiento.

\section{A los padres de familia:}

Mantener una comunicación constante con los responsables de sus instituciones educativas y participar activamente en las actividades sugeridas en los programas educativos. 


\section{A los docentes:}

Identificar las situaciones, dificultades y necesidades educativas de los padres y madres de familia frente a la coyuntura de la pandemia para que de esta forma se establezcan propuestas de orientación y consejería.

\section{A los especialistas en sistemas e informática:}

Asumir retos y trabajar de forma multidisciplinaria con otras especialidades, en este caso el educativo, mostrando las bondades que ofrecen las tecnologías de la información y comunicaciones como alternativa de solución a problemas sociales.

\section{Referencias bibliográficas}

Abufhele, M., y Jeanneret, V. (2020). Puertas Adentro: La otra cara de la pandemia. Revista Chilena de Pediatría, 91(3). https://scielo.conicyt.cl/scielo.php?script=sci_arttext\&pid=S0370-41062020000300319

ANAR. (2020, 17 de marzo). Consejos de Fundación ANAR para padres y madres ante el Coronavirus. Ayuda a Niños y Adolescentes en Riesgo. https://www.anar.org/wpcontent/uploads/2020/03/Consejos-de-ANAR-para-padres-y-madres-ante-elCoronavirus.pdf

Bossolasco, M., Martín, M., Ruiz, M. y Sabulsky, G. (2020). El encuentro virtual como espacio de aprendizaje: análisis de experiencias a través de videoconferencias. https://www.researchgate.net/publication/340137740_El_encuentro_virtual_como_espaci o_de_aprendizaje_analisis_de_experiencias_a_traves_de_videoconferencias

CEPAL, N. (2020). La educación en tiempos de la pandemia de COVID-19. https://repositorio.cepal.org/bitstream/handle/11362/45904/S2000510_es.pdf?sequence=1 \&isAllowed $=\mathrm{y}$

Delgado, P. (2020, 23 de junio). Aprendizaje sincrónico y asincrónico: definición, ventajas y desventajas. Observatorio de Innovación Educativa del Tecnológico de Monterrey. https://observatorio.tec.mx/edu-news/aprendizaje-sincronico-y-asincronico-definicion

Fresnillo, V., Fresnillo, R., y Fresnillo, M. (2000). Escuela de padres. https://orientacionandujar.files.wordpress.com/2009/05/escuela-de-padres.pdf

García, A.y Cuellar, I. (2020). Impacto psicológico del confinamiento en la población infantil y como mitigar sus efectos: revisión rápida de la evidencia. Asociación Española de Pediatría.

https://reader.elsevier.com/reader/sd/pii/S1695403320301673?token=F3923B0C5B7DBF 793383AD2A007D59543A4CDDE4A4A09226BEAFA0A3256646AFF863BCF4F5519 DD9EB42F9E8659AA9E3

Gómez, L. (2019). Desarrollo de las competencias parentales en las escuelas de padres para mejorar el rendimiento escolar de los estudiantes del nivel secundario en un colegio privado de Lima (trabajo de suficiencia profesional de pregrado). Universidad Antonio Ruiz De Montoya. http://repositorio.uarm.edu.pe/handle/UNIARM/1994 
Lamí, L., Pérez, M., y Rodríguez, M. (2017). Las herramientas de comunicación sincrónica y asincrónica en la clase presencial. Revista Conrado, 12(56). https://conrado.ucf.edu.cu/index.php/conrado/article/view/408

Méndez, C. y Näslund, E. (2020, 15 de junio). La opinión de más de 8,000 familias sobre la educación remota durante la pandemia. Enfoque Educación. https://blogs.iadb.org/educacion/es/opinieducacionremota/\#_ftn1

Montes, T., Trujillo, A., Juárez, C., Mejía, A. y Corro, C. (2020). Plataforma tecnológica de orientación para el fortalecimiento de la resiliencia de estudiantes. Una propuesta para el plantel "Lic. Adolfo López Mateos" de la Universidad Autónoma del Estado de México. Revista Dilemas contemporáneos: Educación, Política y Valores. http://www.dilemascontemporaneoseducacionpoliticayvalores.com/index.php/dilemas/art icle/view/2328

Nuño, B., Álvarez, J., Madrigal, E., Martínez, B., y Miranda, R. (2006). Efectos a corto plazo de un programa educativo "Escuela para padres" sobre el ambiente familiar. Revista Médica del Instituto Mexicano del Seguro Social, 44(6), 519-527. https://www.redalyc.org/pdf/4577/457745536005.pdf

Picón, G., González, G., y Paredes, J. (2020). Desempeño y formación docente en competencias digitales en clases no presenciales durante la pandemia COVID-19. https://preprints.scielo.org/index.php/scielo/preprint/view/778/1075

Pinchak, C. (2020). Pandemia por coronavirus (COVID-19); sorpresa, miedo y el buen manejo de la incertidumbre en la familia. Archivos de Pediatría del Uruguay, 91(2), 76-77. http://www.scielo.edu.uy/pdf/adp/v91n2/1688-1249-adp-91-02-76.pdf

Romero, M. (2005). Escuela de padres. Educación y futuro: revista de investigación aplicada y experiencias educativas, (12), 41-50. https://dialnet.unirioja.es/servlet/articulo?codigo=2047041

Sampieri, R., Fernández, C. y Baptista, P. (2014). Metodología de la Investigación, (6 ed.). Editorial MC GRAW HILL

Unicef. (2020). COVID-19: Seminarios virtuales para la familia. Consejos y guías en tiempos de coronavirus. Unicef Perú. https://www.unicef.org/peru/coronavirus/covid-19-seminariosvirtuales-para-familias-casa

Unicef. (2020). Situación de familias con niños, niñas y adolescentes durante el COVID-19 en

Unicef

Panamá. https://www.unicef.org/panama/media/2601/file/Encuesta\%20de\%20Hogares.pdf

Zuleta, A. (2020). La vida en tiempos de pandemia. Revista Investigium IRE Ciencias Sociales y Humanas, $11(1)$ 7-10. https://investigiumire.unicesmag.edu.co/index.php/ire/article/view/324/362

Barreto, H. (2020). Educación emocional para padres con hijos en edad preescolar mediante metodología activa: propuesta de un diseño de intervención (tesis de maestría). https://reunir.unir.net/handle/123456789/9987 